\title{
Evaluation of Glucomannan Powder as an Ultrasound Transmission Gel Alternative for Resource-Constrained Environments: A Prospective, Comparative Study
}

\author{
Sandra C. Milton, DSc, PA-C; Aaron J. Cronin, DSc, PA-C; Jonathan D. Monti, DSc, PA-C \\ From the Department of Emergency Medicine, Madigan Army Medical Center, Tacoma, WA.
}

\begin{abstract}
Introduction-Resource and logistical constraints may limit the availability of commercial ultrasound (US) transmission gel (USTG) in austere environments. Glucomannan powder, a dietary fiber supplement, can be mixed with tap water to form a gel that may be a field-expedient substitute for USTG. We compared glucomannan gel with a commercial USTG for US image adequacy and quality.

Methods-A single clinician obtained 193 US video clips from 14 different examinations on live-tissue and simulation training models using both commercial and glucomannan USTGs. Four US fellowship-trained providers, blinded to type of gel used, independently reviewed the randomized US video clips. The primary outcome of US image adequacy was scored as "yes" or "no" and analyzed using Pearson $\chi^{2}$ analysis. The secondary outcome of image quality was rated on a 0 to 5 Likert scale and analyzed with the independent $t$ test.

Results-For US image adequacy, commercial USTG was superior to glucomannan gel $(P=0.042$, 95\% CI: $96.5-96.6 \%$ ), with commercial USTG adequate in 96.6\% of images (375 of 388 "yes"), whereas glucomannan USTG was adequate in $93.5 \%$ (359 of 384 "yes") of images. For US image quality there was no statistically significant difference between the 2 USTGs $(P=0.176,95 \%$ CI: 93.4-93.5\%), with commercial USTG rated at 3.4 \pm 1.0 and glucomannan gel at $3.3 \pm 1.1$.

Conclusion-Despite a high image adequacy rate, glucomannan gel proved inferior to commercial USTG for US image adequacy but produced equivalent image quality. Glucomannan USTG may be a reasonable substitute when commercial USTG is unavailable.
\end{abstract}

Keywords: austere, point-of-care, POCUS

\section{Introduction}

Ultrasound (US) is a valuable diagnostic adjunct for medical personnel operating in resource-constrained environments. Recent technological advances, resulting in highly

This work was presented at the 2018 Special Operations Medical Association Scientific Assembly, May 13-17, Charlotte, NC; the 2018 Washington American College of Emergency Physicians Summit, May 31-June 1, Seattle, WA; the Madigan Army Medical Center Research Assembly, May 4, 2018, Tacoma, WA; the Society of Emergency Medicine Physician Assistants Annual Conference, May 3-8, 2018, San Antonio, TX; and the 2018 Military Health System Research Symposium, August 20, 2018, Kissimmee, FL.

Corresponding author: Jonathan D. Monti, DSc, PA-C, RDMS, Department of Emergency Medicine, Madigan Army Medical Center, Tacoma, WA.; e-mail: fugazi44jdm@gmail.com.

Submitted for publication October 2017.

Accepted for publication June 2018. portable US machines and transducers, have enabled its application in remote environments. ${ }^{1-4}$ In addition to increased portability, improvements in US technology have prompted a growing number of healthcare providers to use this imaging modality at the point of care (POC). ${ }^{5}$ US technology requires an effective transmission medium between the transducer and patient to acquire technically adequate images. ${ }^{6-12}$ Commercially available US transmission gel (USTG) can be transported into resource-constrained civilian and military environments, but the quantity may be insufficient because of its relative bulk and limited cargo space. Furthermore, commercial USTG may not be available in remote settings as a result of logistical, financial, and environmental factors. ${ }^{6,7,12}$

Previous studies examined potential substitutes for commercial USTG. Researchers evaluated items commonly 
available in hospitals (eg, hand sanitizer, normal saline, dextrose $5 \%$ in water, and olive oil) and substances that may be carried or resourced in austere settings (eg, cornstarch, cassava root, and bula flour). ${ }^{7-13}$ These alternative USTGs showed varying degrees of promise but were limited by liquid bulk, ease of manufacture, or worldwide availability. ${ }^{6,7,12}$ A recently published descriptive paper suggests water-constituted glucomannan powder may be a suitable USTG for military operations. ${ }^{6}$ Glucomannan powder is a soluble fiber derived from the roots of the Amorphophallus konjac plant, which is widely cultivated in numerous climates and continents and commercially available in many health food and Internet-based stores as a dietary supplement intended for human ingestion. ${ }^{14}$ Glucomannan powder can be carried in lightweight, waterproof plastic bags and easily constituted with tap water as needed at the time of examination. Although suggested as an ideal alternative to commercially produced USTG in remote environments, only anecdotal data were offered to support this claim. This study aimed to prospectively compare glucomannan gel with a commercial USTG for point-of-care US (POCUS) diagnostic adequacy and image quality.

\section{Methods}

\section{STUDY OVERSIGHT}

The institutional review board at Madigan Army Medical Center, Tacoma, WA, approved this prospective, comparative study. All participants voluntarily consented to partake in the research. All authors have no conflicts to report.

\section{PARTICIPANTS}

Two physicians and 2 physician assistants, each with emergency medicine US fellowship training, volunteered to review US video clips and assess them for diagnostic adequacy and image quality. One emergency medicine, US fellowship - trained and credentialed clinician volunteered to obtain all US video clips evaluated. Four medical technicians between the ages of 18 and $50 \mathrm{y}$, with a body mass index less than $30 \mathrm{~kg} \cdot \mathrm{m}^{-2}$ and with anatomy necessary for complete US examinations, volunteered to be US models for image collection.

\section{MATERIALS}

A Sonosite X-Porte (Bothell, WA) US machine was used for all data collection. The commercial USTG used in our study was Aquasonic 100 (Parker Laboratories, Fairfield, $\mathrm{NJ})$ at room temperature. Study investigators identified an adequate USTG from a mixture of glucomannan powder and water by trialing various volumes and temperatures of each component. The formula used for our study was consistent with a previous anecdotal recommendation of $5 \mathrm{~mL}$ (1 tsp) of glucomannan powder mixed with $237 \mathrm{~mL}$ (8 oz) of water at 15.6 to $18.3^{\circ} \mathrm{C}\left(60-65^{\circ} \mathrm{F}\right)$. US images were obtained on human models and a vascular Blue Phantom training model (BPTM) (part no. BPBV110) with a metallic needle inserted.

\section{DESIGN}

A single clinician performed 14 different POCUS studies (Table 1) on live tissue and simulation models. For each US examination, the commercial USTG was used first. The model then was cleaned, and the examination was repeated with the glucomannan USTG. The clinician obtained the best US images possible by employing the optimal examination software and transducer, while independently adjusting for depth and gain. The clinician obtained 28 4-s US video clips (14 with each gel) in 7 series for a total of 193 video clips-3 clips were unintentionally not collected. The clinician recorded US video clips of each examination without identifying which of the 2 USTGs was employed. Study investigators downloaded the US video clips from the Sonosite Xporte and labeled and randomized them with a 4-character alphanumeric code generated by software available at www.random.org. The clips were subsequently incorporated into a Microsoft PowerPoint presentation for viewing. Six video clips were duplicated 3 times throughout the randomized slide presentation for a total presentation of 205 US video clips to assess for intrarater reliability. Each slide contained one 4-s US video clip and one 4-character alphanumeric code. Four emergency US fellowship-trained clinicians independently evaluated the randomized US video clips. Reviewers were blinded to the USTG used and the duplicated video clips.

\section{OUTCOME MEASURES}

The primary outcome measurement was US image diagnostic adequacy. For each US video clip, reviewers were asked, "Is this image technically adequate for making a diagnosis or clinical decision?" Results were recorded as a "yes" or "no." The secondary outcome measurement was US image quality. Reviewers were asked to rate the quality of each US image on a 0 to 5 Likert scale based on the resolution. A score of 0 was "unacceptable," and a score of 5 was considered "excellent." For this study, resolution was defined as the reviewer's subjective assessment of the image detail discernible within and between the tissues' inherent echogenicities, irrespective of the predetermined depth, gain, and scan technique. Other secondary outcomes measured were inter- and 
Table 1. Number of ultrasound views obtained and reviewed by anatomical region

\begin{tabular}{|c|c|c|c|c|}
\hline & \multirow[t]{2}{*}{ Collected Images } & \multicolumn{2}{|c|}{ Duplicated Images } & \multirow[t]{2}{*}{ Reviewed Images } \\
\hline & & Gel A & Gel B & \\
\hline \multicolumn{5}{|c|}{ Low-frequency phased array transducer } \\
\hline Aorta & 14 & & & 14 \\
\hline GB-long & $12 *$ & & & 12 \\
\hline Bladder & 14 & & & 14 \\
\hline Cardiac PSL & 14 & & & 14 \\
\hline RUQ & $13^{a}$ & +2 & +2 & 17 \\
\hline Lung & 14 & +2 & +2 & 18 \\
\hline Thoracic & 14 & & & 14 \\
\hline IVC & 14 & & & 14 \\
\hline \multicolumn{5}{|c|}{ High-frequency linear array transducer } \\
\hline GSV & 14 & & & 14 \\
\hline $\mathrm{CFV}$ & 14 & & & 14 \\
\hline Internal jugular & 14 & +2 & +2 & 18 \\
\hline Quad tendon & 14 & & & 14 \\
\hline BPTM-long & 14 & & & 14 \\
\hline BPTM-short & 14 & & & 14 \\
\hline Total & 193 & & & 205 \\
\hline
\end{tabular}

Gel A, commercial ultrasound gel; gel B, glucomannan ultrasound gel.

BPTM, Blue Phantom training model; CFV, common femoral vein; GB, gallbladder; GSV, greater saphenous vein; IVC, inferior vena cava; PSL, parasternal long; Quad, quadriceps; RUQ, right upper quadrant.

${ }^{a}$ The gallbladder was unintentionally missed during the first series with both gels A and B, and the RUQ was missed with gel B during the third series.

intrarater reliability between and among image reviewers. Reviewer scores for diagnostic adequacy and image quality for 18 duplicated US videos were used to calculate these valuations.

\section{STATISTICAL ANALYSIS}

We performed all statistical analysis with IBM SPSS 3.0. We compared study variables between US examinations performed with commercial and glucomannan USTG using the Pearson $\chi^{2}$ test for the primary outcome and independent $t$ test for the secondary outcome. We reported dichotomous variables as proportions and ordinal variables as means with standard deviations. We assessed inter- and intrarater reliability among the reviewers using Cronbach $\alpha$ test and a calculation of intraclass correlation. An $\alpha>0.80$ correlates with good consistency, and an intraclass correlation between 0.60 and 0.75 is considered good agreement.

For prestudy power analysis, using a conventional $\alpha$ of $5 \%$ and $\beta$ of $80 \%$, we estimated the effect size at 0.41 and set the minimal clinically relevant difference at $3 \%$. A sample size of 180 images (90 with each type of USTG) was calculated based on these parameters.

\section{Results}

A total of 193 US video clips were recorded and independently reviewed by 4 emergency US fellowship-trained clinicians (Figure 1). For diagnostic adequacy, commercial USTG was sufficient $96.6 \%$ of the time (375 of 388 "yes"), and glucomannan USTG was adequate in $93.5 \%$ of its utilizations (359 of 384 "yes") (Table 2). Commercial USTG was superior to glucomannan gel for diagnostic adequacy $(P=0.042$, 95\% CI: 96.5-96.6\%). For image quality, commercial USTG was rated at $3.4 \pm 1.0$ and glucomannan gel at $3.3 \pm 1.1$, indicating similar efficacy $(P=0.176,95 \%$ CI: 93.4-93.5\%) (Figure 2). For interrater reliability, a mean Cronbach $\alpha$ of 0.837 indicated good consistency between individual reviewers (Table 3). Intrarater reliability could not be calculated with the available data; a total of 9 images required duplication to calculate statistically significant reliability (Table 4).

\section{Discussion}

We compared the quality and adequacy of glucomannan USTG-obtained images vs commercial USTG-obtained images when performing POCUS examinations. Glucomannan USTG performed as well as the commercial USTG with respect to image quality. With respect to diagnostic adequacy, however, commercial USTG was found 
a

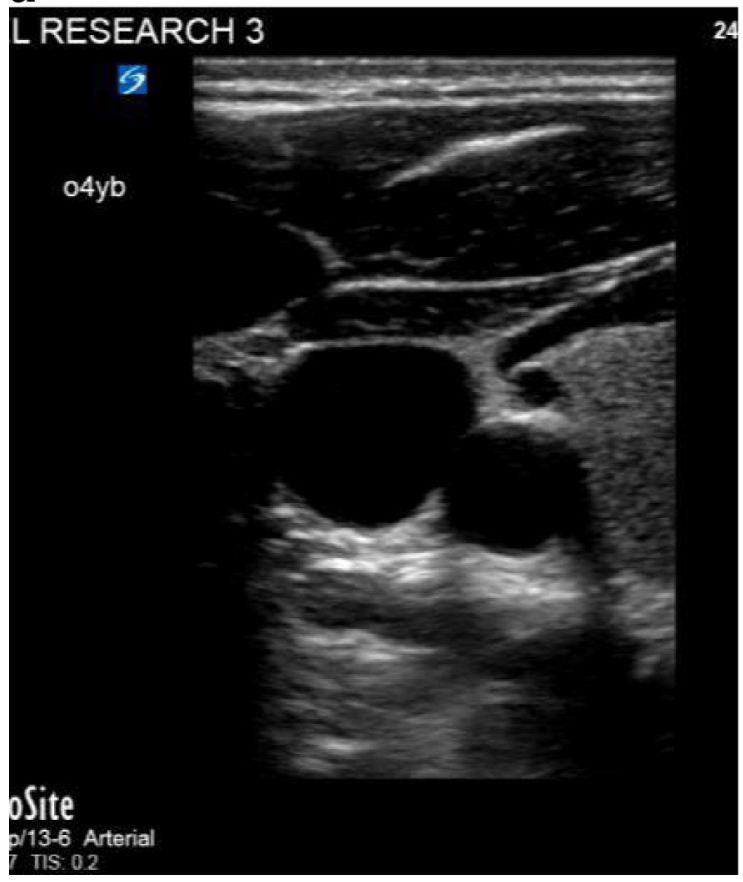

b

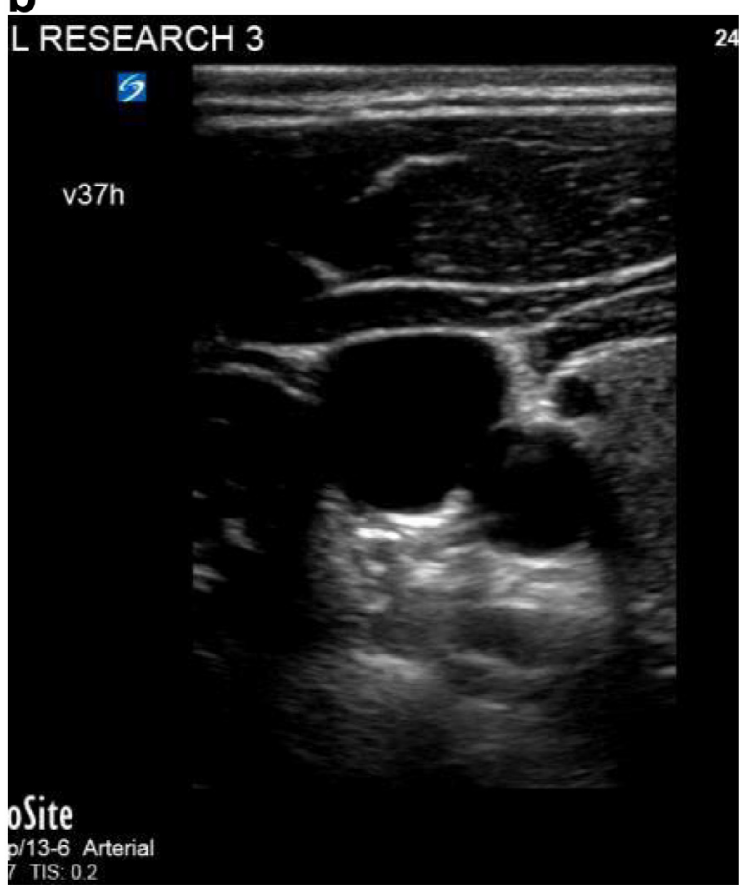

Figure 1. Internal jugular vein imaged with commercial (a) and glucomannan gels (b).

Table 2. Results of primary and secondary outcomes

\begin{tabular}{lccr}
\hline & $\begin{array}{c}\text { Commercial } \\
\text { ultrasound gel }\end{array}$ & $\begin{array}{c}\text { Glucomannan } \\
\text { ultrasound gel }\end{array}$ & Difference \\
\hline Adequacy (yes): \% (95\% CI) & $96.6(96.5-96.6)$ & $93.5(93.4-93.5)$ & $3.1 \%$ \\
Quality (0-5 scale): mean score (95\% CI) & $3.4(3.3-3.5)$ & $3.3(3.2-3.4)$ & 0.1 \\
\hline
\end{tabular}

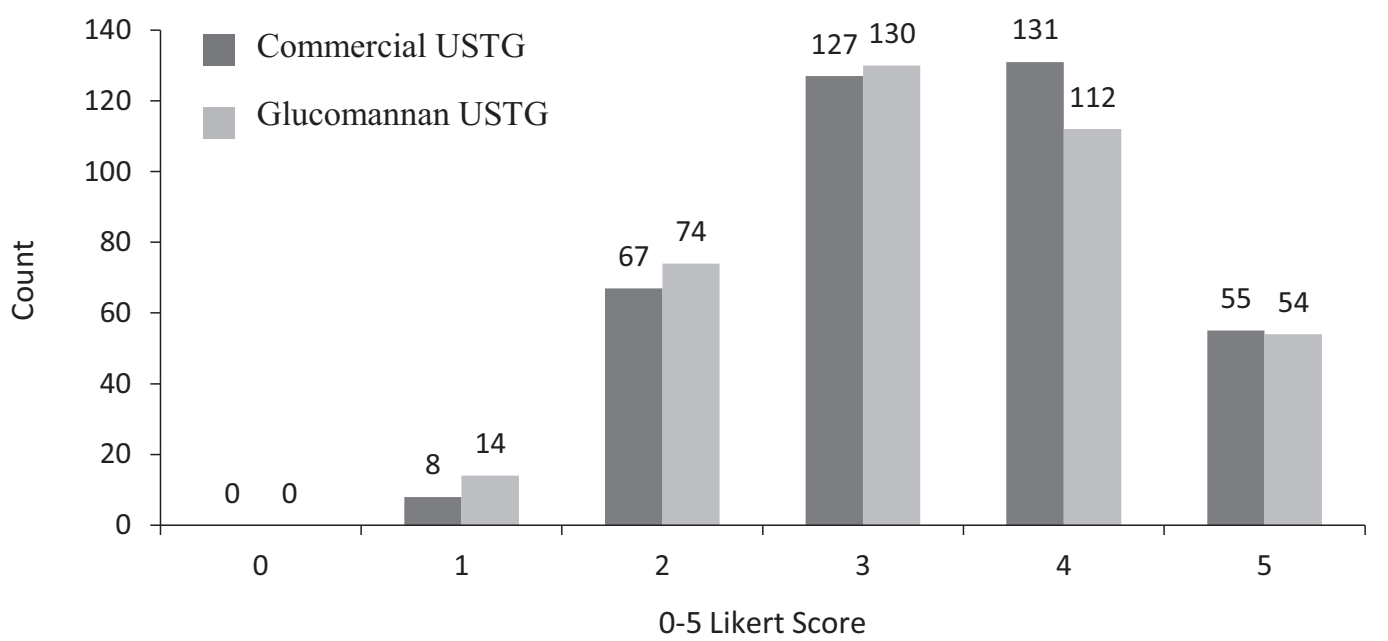

Figure 2. Number of 0 to 5 Likert scores for ultrasound image quality received for each ultrasound transmission gel (USTG) ( $n=772$ ). 
Table 3. Interrater reliability of ultrasound video clip reviewers

\begin{tabular}{llll}
\hline Groups & Cronbach $\alpha$ & Reviewer & $\begin{array}{l}\text { Intraclass } \\
\text { correlation }\end{array}$ \\
\hline
\end{tabular}

All 4 clip reviewers

Reviewers 2, 3, 4

Reviewers 1, 3, 4

Reviewers 1, 2, 4

Reviewers 1, 2, 3
0.837

0.786

0.776

0.788

0.826
0.550

0.535

0.553

0.613 to be slightly superior to glucomannan USTG. We found interrater reliability was consistent, with good agreement among the image reviewers. Our findings suggest glucomannan powder may be a suitable USTG substitute when commercial USTG is not available.

Because of the subjectivity often associated with review of US images, we compared reviewer scores for embedded duplicate video clips to evaluate inter- or intrarater reliability. Previously published data on USTG substitutes did not report rater reliability scores. ${ }^{7,8,10,12}$ One study screened reviewers with a "pretest" composed of "conventionally acquired" US images of varying quality. ${ }^{8}$ The goal of the pretest was to determine a numerical threshold of minimal acceptable quality, not to assess for reviewer reliability. In our study we found that interrater reliability was sufficiently high. This finding may be explained by our reviewers having similar levels of US training and practical application of POCUS. Previous studies incorporated radiologists as reviewers, who may be more accustomed to the higher quality imaging typically produced by large, hospital-based US machines not designed for POCUS use. ${ }^{7,8}$ Future research evaluating alternative USTGs should consider including interrater analyses because US image interpretation is reviewer dependent.
Our study found image quality for both USTGs was similar for a comprehensive set of US examinations. The variety of body areas scanned in this study included shallow and deep anatomical structures that require imaging with both high- and low-frequency transducers, representing a wide range of examinations commonly obtained in the acute setting. Additionally, we incorporated a metallic foreign body in the BPTM to permit evaluation of reverberation and US image artifact with both the commercial and glucomannan USTG. Placing a needle in the BPTM partially represented US-guided procedures, such as central venous access, regional anesthesia, intra-articular infusions, and so on.

Ocular and obstetrical studies were considered but omitted for safety reasons. The safety of glucomannan gel in the eye has not been tested, and exposure of a developing fetus to unnecessary US was not required to evaluate the objectives of this study.

Previously published data support multiple other substitutes for commercial USTG. One study found that olive oil had no statistical difference compared with commercial USTG when evaluating sonographic image quality, and the medium was preferred by the 346 patients participating in the study. ${ }^{10}$ Another report found that olive oil, along with hand sanitizer and hair styling gel, yielded image qualities similar to that of commercial USTG. Hand lotion, liquid detergent, and baby shampoo scored comparatively lower as alternative USTGs but were considered acceptable alternatives. ${ }^{8}$ Hand sanitizer is recommended as an USTG alternative for peripheral nerve blocks, with the dual benefit of sterilizing the skin and costing less than sterile USTG packets and chlorhexidine preparation swabs. ${ }^{9}$ Dextrose $5 \%$ in water $\left(\mathrm{D}_{5} \mathrm{~W}\right)$ is also recommended for peripheral nerve blocks because of superior probe stability when the $\mathrm{D}_{5} \mathrm{~W}$ evaporates and creates friction, while still achieving an excellent US image. ${ }^{13}$ For the same reasons, normal saline is reported

Table 4. Image reviewers' scores for 3 viewings of each duplicated ultrasound image

\begin{tabular}{|c|c|c|c|c|c|c|c|c|c|}
\hline \multirow[b]{2}{*}{ Image } & \multirow[b]{2}{*}{ Gel } & \multicolumn{4}{|c|}{$\begin{array}{l}\text { Diagnostic technical adequacy? (Yes/No) } \\
\text { Image reviewer }\end{array}$} & \multicolumn{4}{|c|}{$\begin{array}{l}\text { Image quality }(0-5) \text { Likert scale } \\
\text { Image reviewer }\end{array}$} \\
\hline & & 1 & 2 & 3 & 4 & 1 & 2 & 3 & 4 \\
\hline Jugular & A & $Y-Y-Y$ & Y-Y-Y & $Y-Y-Y$ & $Y-Y-Y$ & $5-5-5$ & $5-4-4$ & $3-4-3$ & $4-4-3$ \\
\hline Jugular & $\mathrm{B}$ & Y-Y-Y & Y-Y-Y & $Y-Y-Y$ & Y-Y-Y & $5-4-5$ & $4-5-5$ & $4-3-3$ & $5-4-3$ \\
\hline Lung & A & Y-Y-Y & Y-N-Y & $Y-Y-Y$ & $Y-Y-Y$ & $3-4-4$ & $2-4-3$ & $2-2-2$ & $2-3-2$ \\
\hline Lung & $\mathrm{B}$ & $Y-Y-Y$ & $Y-Y-Y$ & $Y-Y-Y$ & $Y-Y-Y$ & $5-4-4$ & $3-3-3$ & $3-3-3$ & $3-3-3$ \\
\hline RUQ & A & Y-Y-Y & $Y-Y-Y$ & $Y-Y-Y$ & Y-Y-Y & $5-4-3$ & $5-3-4$ & $4-3-3$ & $3-3-3$ \\
\hline RUQ & $\mathrm{B}$ & Y-Y-Y & Y-Y-Y & $Y-Y-Y$ & Y-Y-Y & $4-4-3$ & $5-4-4$ & $4-3-2$ & $3-3-3$ \\
\hline
\end{tabular}

gel A, commercial USTG; gel B, glucomannan USTG.

N, no; RUQ: right upper quadrant; USTG, ultrasound transmission gel; Y, yes. 
as an alternative USTG medium for internal jugular venous cannulation. ${ }^{11}$ For resource-constrained environments, one study suggested widely available cornstarch mixed with boiling water as an ideal USTG alternative. ${ }^{7}$ Another paper found that cassava root and bula flour, available in local markets in Africa, proved to be reliable USTG alternatives. ${ }^{12}$ None of these published reports, however, evaluated glucomannan powder.

Glucomannan powder may be a superior alternative for military operations and humanitarian missions in resourceconstrained environments. This is especially pertinent in regions such as the South Pacific, southwest Asia, and Africa where $A$ konjac is both indigenous and farmed. ${ }^{14}$ Hand sanitizer and olive oil are gel/liquid mediums with relatively larger volumes and weights that make them as logistically cumbersome as commercial USTG. These disadvantages should be considered when developing load plans for austere environments. Also, hand sanitizer is alcohol based, may evaporate rapidly, and possibly requires a greater total volume to complete the US examination. Cornstarch, cassava root, and bula flour, on the other hand, are powders and offer the advantages of low weight and volume. However, each requires the solution to be boiled to produce the gel, which may not be feasible or timely when evaluating patients at the POC in austere settings. Glucomannan powder can be carried in lightweight, waterproof plastic bags and is easily constituted with tap water without requiring a heat source. It may be mixed as needed at the time of examination in a relatively short time and at a relatively low cost.

Analyses have found commercial USTG to be relatively cost prohibitive compared with recommended USTG alternatives (Table 5). 6,7,10,12 Glucomannan powder is routinely sold in $227-\mathrm{g}$ bottles costing US $\$ 11$ to $\$ 15$, with the ability to produce nearly 500.25 -L aliquots of USTG. ${ }^{6}$ Cornstarch, cassava, and bula flour are inexpensive but may not be readily available in certain regions of the world. ${ }^{7,12}$ Hand sanitizer used in place of a sterile USTG single-use packet and chlorhexidine skin preparation swabs resulted in a $6000 \%$ cost savings for US-guided procedures. ${ }^{9}$ Overall, all previously

Table 5. Cost comparison of ultrasound gels

\begin{tabular}{ll}
\hline Ultrasound transmission gel & Cost per 0.25 L \\
\hline Commercial & $\$ 1-\$ 5$ \\
Glucomannan $^{6}$ & $\$ 0.30$ \\
Cornstarch $^{l}$ & $<\$ 0.10$ \\
Cassava flour $^{9}$ & $\$ 0.09$ \\
Bula & $\$ 0.25$ \\
Olive oil & 4 \\
\hline
\end{tabular}

researched and recommended alternative USTGs offer a cost benefit over commercial USTG.

Several published articles have highlighted contamination of multiuse commercial USTG bottles and associated nosocomial infections, including a class I Food and Drug Administration recall of nonsterile Sonic Generic USTG in 2012 because of the presence of Pseudomonas aeruginosa and Klebsiella oxytocan. ${ }^{15-22}$ Glucomannan gel may be a medium for bacterial growth because there are no known preservatives. Glucomannan USTG is intended to be constituted at the POC and discarded after the US examination is complete. Rapid application of glucomannan USTG after constitution may not permit bacterial growth. However, we do not recommend performing USguided tissue- or mucus membrane-invasive procedures before the completion of formal research evaluating bacterial contamination and growth in glucomannan USTG.

Our study has several important limitations. Five of the 14 anatomical regions in this study included the abdomen on live-tissue models. Movement of air through the intestines is a known source of artifact in US images; with constant peristalsis of the intestines, it is impossible to obtain exact views of abdominal organs with different gels. However, this same limitation likely applied to previous, similar US studies that used live-tissue models. Another limitation was that all live-tissue models had a body mass index less than $30 \mathrm{~kg} \cdot \mathrm{m}^{-2}$. This limits the generalizability of our results to obese patients. All US video clips were obtained by a single clinician who was not blinded to the type of USTG used because of differences in color and consistency of the gels. Therefore we cannot exclude the possibility of preference bias and its influence on recorded images. Lastly, we did not re-create the remote environment in which glucomannan USTG may be used. Precise measurement tools were used to prepare the glucomannan gel, and a hospital-quality POCUS machine and transducers were used. Lower-quality US machines and transducers may be all that is available in the resourceconstrained environment and may correspondingly degrade US image quality.

\section{Conclusions}

Glucomannan USTG proved inferior to commercial USTG for US image adequacy but produced equivalent image quality. Glucomannan USTG, because of its comparative benefits in terms of bulk, cost, and ease of manufacture at the POC, may be a useful and reasonable substitute when commercial USTG is unavailable, particularly for those operating in unconventional environments. Although additional research is needed to further investigate glucomannan, its benefits for those operating in unconventional settings may outweigh its limitations. 
Acknowledgments: The authors acknowledge members of Madigan Army Medical Center's Department of Clinical Investigation. In particular, they sincerely thank Melissa Borsic, Athena Rayner, and Barbara Jones for their expertise in navigating the institutional review board process, Dr Raywin Huang for his assistance with statistical analysis, Jodi Quesnell for her performance of prestudy literature searches, and MAJ Jason Naylor for his assistance with manuscript revision.

Author Contributions: Study concept and design (SCM, AJC, JDM); acquisition of data (SCM, AJC); analysis of data (SCM, AJC); drafting of manuscript (SCM, AJC, JDM); critical revision of manuscript (SCM, AJC, JDM); and approval of final manuscript (SCM, AJC, JDM).

Financial/Material Support: None.

Disclosures: This study was conducted under a protocol reviewed and approved by the Madigan Army Medical Center institutional review board. The views expressed are those of the authors and do not reflect the official policy or position of the US Army Medical Department, Department of the Army, Department of Defense, or the US Government. The authors have no potential commercial and/or professional conflicts of interest to disclose.

\section{References}

1. Russell TC, Crawford PF. Ultrasound in the austere environment: a review of the history, indications, and specifications. Mil Med. 2013;178(1):21-8.

2. Gharahbaghian L, Anderson KL, Lobo V, Huang R-W, Poffenberger CM, Nguyen PD. Point-of-care ultrasound in austere environments: a complete review of its utilization, pitfalls, and technique for common applications in austere settings. Emerg Med Clin North Am. 2017;35(2):409-41.

3. Wagner MS, Garcia K, Martin DS. Point-of-care ultrasound in aerospace medicine: known and potential applications. Aviat Space Environ Med. 2014;85(7):730-9.

4. Nelson BP, Sanghvi A. Out of hospital point of care ultrasound: current use models and future directions. Eur J Trauma Emerg Surg. 2016;42(2):139-50.

5. Moore CL, Copel JA. Point-of-care ultrasonography. $N$ Engl J Med. 2011;364(8):749-57.

6. Monti JD. A novel ultrasound transmission gel for resource-constrained environments. J Spec Oper Med. 2017;17(1):22-5.

7. Binkowski A, Riguzzi C, Price D, Fahimi J. Evaluation of a cornstarch-based ultrasound gel alternative for lowresource settings. J Emerg Med. 2014;47(1):e5-9.

8. Savaser D, Gray S, Medak A, Shah V, Allen D, Castillo E. The USAGES study (Ultrasonographic Study of Alternative Gel Experimental Substances): a trial of alternative substances for use in clinical sonographic imaging. Presented at: American Institute Ultrasound in Medicine Annual Convention; AIUM Annual Convention 2013. New York, NY. https://www.aium.org/cme/pastcons/2013finalprogram.pdf. Accessed December 12, 2016.

9. Sutton E, Bullock WM, Khan T, Eng M, Gadsden J. Hand sanitizer as an alternative to ultrasound transmission gel. Reg Anesth Pain Med. 2016;41(5):655-6.
10. Luewan S, Srisupundit K, Tongsong T. A comparison of sonographic image quality between the examinations using gel and olive oil, as sound media. J Med Assoc Thail. 2007;90(4):624-7.

11. Dohgomori H, Shiba M, Okamoto K. Application of normal saline to ultrasonography as an alternative to gel for internal jugular venous cannulation. Can J Anaesth. 2007;54(3):246.

12. Salmon M, Salmon C, Bissinger A, Muller MM, Gebreyesus A, Geremew $\mathrm{H}$, et al. Alternative ultrasound gel for a sustainable ultrasound program: application of human centered design. PloS One. 2015;10(8) e0134332.

13. Tsui BCH. Dextrose $5 \%$ in water as an alternative medium to gel for performing ultrasound-guided peripheral nerve blocks. Reg Anesth Pain Med. 2009;34(5):525-7.

14. Hetterscheid W, Ittenbach S. Everything you always wanted to know about Amorphophallus, but were afraid to stick your nose into!!!!!. Aroideana. 1996;19:7-131.

15. American College of Emergency Physicians. Ultrasound guidelines: emergency, point-of-care, and clinical ultrasound guidelines in medicine [policy statement]. Approved June 2016. https://www.acep.org/globalassets/uploads/uploadedfiles/acep/membership/sections-of-membership/ultra/ultra sound-policy-2016-complete_updatedlinks_2018.pdf.

Accessed Jan 20, 2017.

16. Centers for Disease Control and Prevention (CDC). Pseudomonas aeruginosa respiratory tract infections associated with contaminated ultrasound gel used for transesophageal echocardiography-Michigan, December 2011-January 2012. MMWR Morb Mortal Wkly Rep. 2012;61:262-4.

17. Jacobson M, Wray R, Kovach D, Henry D, Speert D, Matlow A. Sustained endemicity of Burkholderia cepacia complex in a pediatric institution, associated with contaminated ultrasound gel. Infect Control Hosp Epidemiol. 2006;27(4):362-6.

18. Spratt HG, Levine D, Tillman L. Physical therapy clinic therapeutic ultrasound equipment as a source for bacterial contamination. Physiother Theory Pract. 2014;30(7):507-11.

19. Provenzano DA, Liebert MA, Steen B, Lovetro D, Somers DL. Investigation of current infection-control practices for ultrasound coupling gel: a survey, microbiological analysis, and examination of practice patterns. Reg Anesth Pain Med. 2013;38(5):415-24.

20. Showronek P, Wojciechowski A, Leszczynski P, Olszewski P, Sibiński M, Polguj M, et al. Can diagnostic ultrasound scanners be a potential vector of opportunistic bacterial infection? Med Ultrason 18(3):326-31.

21. Schabrun S, Chipchase L, Rickard H. Are therapeutic ultrasound units a potential vector for nosocomial infection? Physiother Res Int. 2006;11(2):61-71.

22. US Food and Drug Administration. Class 1 device recall other sonic generic ultrasound transmission gel. Recall event number 61732. Posted May 24, 2012. Accessed Jan 14, 2017 at https://www.accessdata.fda.gov/scripts/cdrh/ cfdocs/cfRES/res.cfm?id=108813. 